\title{
Atom structures and Sahlqvist equations
}

\author{
Yde Venema \\ Department of Mathematics and Computer Science \\ Vrije Universiteit \\ De Boelelaan 1081 \\ 1081 HV Amsterdam
}

July 28, 1997

\begin{abstract}
This paper addresses the question for which varieties of boolean algebras with operators membership of an atomic algebra $\mathfrak{A}$ is determined by its atom structure $\mathfrak{A} \mathfrak{A} \mathfrak{A}$. We prove a positive answer for conjugated Sahlqvist varieties; we also show that the conjugation condition is necessary. As a corollary to the positive result and a recent result by I. Hodkinson, we prove that the variety RRA of representable relation algebras, although canonical, cannot be axiomatised by Sahlqvist equations.
\end{abstract}




\section{Introduction}

Ever since JónsSON \& TARSKI [7] introduced the notion of a boolean algebra with operators (a BAO for short), the relation between atomic BAOs and their atom structures has received attention. This note is concerned with the question, for which varieties of BAOs membership of an atomic BAO $\mathfrak{A}$ is determined by the atom structure of $\mathfrak{A}$. For conjugated BAOs, we can show that any Sahlqvist variety (i.e., a variety defined by Sahlqvist equations) has this property. To be more precise, it is the main aim of the paper to prove the following theorem. Examples of varieties that fall under its scope include relation algebras and cylindric algebras.

Theorem 1 Let $\mathfrak{A}$ and $\mathfrak{B}$ be atomic, conjugated boolean algebras with operators, such that $\mathfrak{A} \mathfrak{t} \mathfrak{A} \cong \mathfrak{A} \mathfrak{\mathfrak { B }}$. Then for every Sahlqvist equation $\eta$ :

$$
\mathfrak{A}=\eta \Longleftrightarrow \mathfrak{B}=\eta .
$$

This result, of which a proof will be supplied in section 3, adds to a list of nice properties of Sahlqvist varieties. For instance, it was already known that any Sahlqvist equation $\eta$ is:

canonical: $\eta$ holds in an algebra $\mathfrak{A}$ iff $\eta$ holds in the canonical embedding algebra (or perfect extension) of $\mathfrak{A}$, and

structure-elementary: there is a first-order formula $\eta^{s}$ in the language of atom structures, which is effectively obtainable from $\eta$, and such that for any relational structure $\mathfrak{F}, \eta$ is valid in the full complex algebra $\mathfrak{C m} \mathfrak{F}$ if and only if $\eta^{s}$ is valid in $\mathfrak{F}$.

Together, these properties imply that any Sahlqvist variety $\mathrm{V}$ is canonical (closed under taking perfect extensions), and that the class Str $\mathrm{V}$ of structures for $\mathrm{V}$ (that is, relational structures $\mathfrak{F}$ whose complex algebra $\mathfrak{C m} \mathfrak{F}$ belongs to $\mathrm{V}$ ) is elementary; in fact, it is axiomatized by a set of first order conditions which can be effectively obtained from the equational axiomatization for $\mathrm{V}$. We refer to DE RiJke \& Venema [2] for more information on Sahlqvist varieties, or to JónSSON [6] for a fully algebraic discussion of their canonicity.

This all combines well with our Theorem 1. From the second property of Sahlqvist equations and Theorem 1 it follows that a Sahlqvist equation $\eta$ holds in an atomic, conjugated BAO $\mathfrak{A}$ if and only if $\eta^{s}$ is true of the atom structure of $\mathfrak{A}$. From this, Corollary 1 below is immediate. Let At $\mathrm{V}$ denote the class of atom structures of atomic algebras in $\mathrm{V}$.

Corollary 1 Let $\mathrm{V}$ be a conjugated Sahlqvist variety of boolean algebras with operators. Then $\mathrm{Str} \mathrm{V}=\mathrm{At} \mathrm{V}$; hence, At $\mathrm{V}$ is an elementary class, axiomatized by a set of conditions which can be effectively obtained from the equational axiomatization for $\mathrm{V}$.

And using the canonicity of Sahlqvist equations, we can easily derive the following corollary to Theorem 1 - its proof is delayed again to section 3 .

Corollary 2 Let $\mathrm{V}$ be a conjugated Sahlqvist variety of boolean algebras with operators. Then $\mathrm{V}=\mathrm{SCm}$ At V. 
Let us agree to call a class $\mathrm{K}$ atom-canonical if $\mathrm{Str} \mathrm{K}=$ At $\mathrm{K}$. This name is explained by the equivalent characterization that for any atomic algebra $\mathfrak{A}$ in $\mathrm{V}$, the complex algebra of its atom structure should be in $V$ as well. Such varieties are investigated in for instance GoLDBLATT [3]; one of the main results of that paper is that any canonical variety with this property is elementarily generated, that is, there is an elementary class $F$ of relational structures such that $\mathrm{V}=\mathrm{HSPCm} F$. Goldblatt shows that atom-canonicity is not a necessary condition for this result: he displays a canonical variety that is not atom-canonical but nevertheless elementarily generated.

We will see in this paper that there are in fact very simple examples of this kind, and thus, very simple examples of varieties that are not atom-canonical. To be more precise, there exist an atomic BAO $\mathfrak{A}$ and a strictly positive equation $\eta$, such that $\mathfrak{A}=\eta$ while $\mathfrak{C} \mathfrak{m} \mathfrak{A t} \mathfrak{A} \mid \neq \eta$. Since $\mathfrak{A}$ and $\mathfrak{C m} \mathfrak{A} \mathfrak{A} \mathfrak{A}$ have the same atom structure, viz., $\mathfrak{A} \mathfrak{t} \mathfrak{A}$, this example shows that we cannot do without the conjugation condition in Theorem 1. It also shows that the variety defined by $\eta$ is not atom-canonical; since $\eta$ is positive (and hence, a Sahlqvist equation), it is immediate that this variety is generated by the elementary class of all structures that satisfy $\eta^{s}$. This example will be treated in detail in section 4, where the notion of atom-canonicity is studied for the general case of not necessarily conjugated BAOs .

First we turn to an application of Theorem 1 in the area of algebraic logic. Consider the variety RRA of representable relation algebras - for definitions and some basic theory concerning (representable) relation algebras the reader is referred to MADDUX [8].

In Hodkinson [5] an example is given of two atomic relation algebras $\mathfrak{A}$ and $\mathfrak{C m} \mathfrak{A} \mathfrak{A}$ of which only $\mathfrak{A}$ is representable. ${ }^{1}$ Note that since relation algebras are conjugated, this implies that not every conjugated canonical variety is atom-canonical. Also, from the result of Hodkinson and Theorem 1 the following is immediate.

Corollary 3 The variety RRA cannot be axiomatized by Sahlqvist equations.

This may be of interest for two reasons. First, from results in Monk [9] it has been known for a long time that RRA does not allow a finite axiomatization. Monk's result has been considerably strengthened by others, to the effect that any equational axiomatization of RRA must have a rather complex structure, see for instance ANDRÉKA [1]. Corollary 3 further lowers one's expectations concerning the mathematical transparancy of any equational axiomatization for RRA; it also generalizes a result by Andréka that RRA is not axiomatizable by positive equations. Second, Corollary 3 relates to an open problem in the area of relation algebras, namely, whether the class StrRRA is elementary — or, equivalently, whether it is closed under ultraproducts. (These questions are equivalent since any class of the form Str V is closed under ultraroots.) For, if RRA were a Sahlqvist variety, the earlier mentioned fact that Sahlqvist equations are structure-elementary would immediately answer the question to the positive. Now of course, Corollary 3 in itself does not provide a negative answer to the problem, but it does give some evidence that a positive answer may be hard to find.

\footnotetext{
${ }^{1}$ In the most recent version of the paper, Hodkinson has transferred his results to finite-dimensional representable cylindric algebras. Hence, it follows from these new results of Hodkinson and Theorem 1 that for each $n \geq 3$, the variety $\mathrm{RCA}_{n}$ is not axiomatizable by Sahlqvist equations.
} 
Some results related to this paper can be found in Venema [13]; the main result of that paper states that the class At $\mathrm{V}$ is elementary for any variety $\mathrm{V}$ that is conjugated. Finally, there are interesting connections between the results reported on in this paper and the notion of the completion a boolean algebra with operators as defined in MONK [10]. (This connection was also pointed out by S. Givant.) We hope to report on this connection in a future publication.

Acknowledgements The research of the author has been made possible by a fellowship of the Royal Netherlands Academy of Arts and Sciences and a visiting fellowship of Victoria Univerity Wellington. A first version of this paper appeared as VENEMA [12]. Thanks are due to Rob Goldblatt for discussions and for making the visit to Wellington possible. Finally, I am grateful to the anonymous referee for a number of very helpful comments.

\section{Terminology and notation}

We understand familiarity with boolean algebras and some standard notions related to them, such as atomicity or infinite joins. The power set of a set $W$ is denoted by $\mathcal{P}(W)$, the power set algebra by $\mathfrak{P}(W)$.

An operation on a boolean algebra $\mathfrak{A}=(A,+,-, 0)$ is nothing but a function $f: A^{n} \rightarrow A$ for some $n \in \omega$. The dual of an operation $f: A^{n} \rightarrow A$ is defined as $f_{\delta}\left(a_{1}, \ldots, a_{n}\right)=$ $-f\left(-a_{1}, \ldots,-a_{n}\right)$. An operation $f$ is normal if $f\left(a_{1}, \ldots, a_{n}\right)=0$ whenever $a_{i}=0$ for one of the arguments $a_{i}$; additive if it is join-preserving in each of its arguments; completely additive if it preserves arbitrary joins in each of its arguments; and monotonic if it is increasing in each of its arguments. In this paper, an operation will be called an operator if it is normal and additive (it is customary to require additivity only).

Two unary operations $f$ and $g$ on $\mathfrak{A}$ are called conjugates if for all $a, b$ in $A$ it holds that $a \cdot f(b)=0$ iff $g(a) \cdot b=0$. An equivalent characterization is that $a \leq f_{\delta}(b)$ iff $g a \leq b$ for all $a$ and $b$. The notion of conjugation extends to operations of arbitrary rank, but we only treat the binary case here: three binary operations $f_{1}, f_{2}$ and $f_{3}$ are called conjugates if for all $a_{1}$, $a_{2}$ and $a_{3}$, we have: $a_{1} \cdot f_{1}\left(a_{2}, a_{3}\right)=0$ iff $a_{2} \cdot f_{2}\left(a_{3}, a_{1}\right)=0$ iff $a_{3} \cdot f_{3}\left(a_{1}, a_{2}\right)=0$. Conjugation can also be expressed equationally (for unary operations, two axioms suffice: $f g_{\delta} x \leq x$ and $\left.g f_{\delta} x \leq x\right)$. A BAO is conjugated if for each of its operators there are conjugates in the clone of operations generated by the basic operations; for reasons of notational simplicity, in this paper we always assume to be dealing with the special case in which the operators themselves already come in conjugated tuples.

A similarity type is a pair $\tau=(I, \rho)$ such that $I$ is a set of operation symbols and $\rho: I \rightarrow \omega$ is a map assigning to each operation symbol a finite rank. A boolean algebra with $\tau$-operators, short: a $\tau$-BAO, is an algebra $\mathfrak{A}=\left(A,+,-, 0, f_{i}\right)_{i \in I}$ such that each $f_{i}$ is a $\rho(i)$-ary operator on the boolean algebra $(A,+,-, 0)$. A relational $\tau$-structure is a structure $\mathfrak{F}=\left(W, T_{i}\right)_{i \in I}$ such such that each $T_{i}$ is a $\rho(i)+1$-ary relation on $W$; elements of $W$ will be called states of $\mathfrak{F}$. Notions concerning atoms pertain to a BAO as to its underlying boolean algebra; the set of atoms of an atomic BAO $\mathfrak{A}$ is denoted by At $\mathfrak{A}$. Given an $n$-ary operator $f$ on the atomic 
boolean algebra $\mathfrak{A}$, the $n+1$-ary relation $R_{f}$ on $A t \mathfrak{A}$ is defined by ${ }^{2}$

$$
R_{f} a b_{1} \ldots b_{n} \text { iff } a \leq f\left(b_{1}, \ldots, b_{n}\right) .
$$

The atom structure of the atomic $\tau$-BAO $\mathfrak{A}=\left(A,+,-, 0, f_{i}\right)_{i \in I}$ is the $\tau$-structure $\mathfrak{A} \mathfrak{t} \mathfrak{A}=$ $\left(A t \mathfrak{A}, R_{f_{i}}\right)_{i \in I}$. The canonical structure of a $\tau$-BAO $\mathfrak{A}=\left(A,+,-, 0, f_{i}\right)_{i \in I}$ is defined as the $\tau$-structure $\mathfrak{C}_{\mathfrak{s}} \mathfrak{A} \mathfrak{t}=\left(U f \mathfrak{A}, Q_{f_{i}}\right)_{i \in I}$, where $U f \mathfrak{A}$ is the set of ultrafilters of (the boolean reduct of) $\mathfrak{A}$, and $Q_{f_{i}}$ is defined by

$$
Q_{f_{i}} u u_{1} \ldots u_{n} \text { iff } f_{i}\left(b_{1}, \ldots, b_{n}\right) \in u \text { whenever } b_{1} \in u_{1}, \ldots, b_{n} \in u_{n} .
$$

Conversely, an $n+1$-ary relation $T$ on a set $W$ induces an $n$-ary operation $m_{T}$ on $\mathcal{P}(W)$ :

$$
m_{T}\left(X_{1}, \ldots, X_{n}\right)=\left\{w \in W \mid T w w_{1} \ldots w_{n} \text { for some } w_{1} \in X_{1}, \ldots, w_{n} \in X_{n}\right\} .
$$

Then, given a relational $\tau$-structure $\mathfrak{F}=\left(W, T_{i}\right)_{i \in I}$, the full complex algebra $\mathfrak{C m} \mathfrak{F}$ of $\mathfrak{F}$ is defined as the power set algebra $\mathfrak{P}(W)$ endowed with an operation $m_{T_{i}}$ for each relation $T_{i}$. A complex algebra is just any subalgebra of a full complex algebra. Such operations $m_{T}$ are always completely additive operators; hence, every complex algebra is a BAO. The canonical embedding algebra of a BAO $\mathfrak{A}$ is the algebra $\mathfrak{C} \mathfrak{m} \mathfrak{C} \mathfrak{A}$.

Given a class $\mathrm{K}$ of $\tau$-BAOs, we define At $\mathrm{K}$ as the class of atom structures of atomic algebras in K. Similar, or familiar definitions apply to the class operations $\mathrm{Cm}, \mathrm{H}, \mathrm{S}$ and $\mathrm{P}$. $\operatorname{Str} \mathrm{K}:=\{\mathfrak{F} \mid \mathfrak{C m} \mathfrak{F} \in \mathrm{K}\}$ is the class of structures for $\mathrm{K}$.

Now we turn to the algebraic language to describe $\tau$-BAOs. Besides the boolean symbols, this language has a $\rho(i)$-adic function symbol for each element $i$ of $I$. We may write $f_{\mathfrak{A}}$ for the interpretation of the function symbol $f$ in the algebra $\mathfrak{A}$, but usually we will be sloppy concerning the distinction between symbols and their interpretations. From these symbols and a set of variables, $\tau$-terms and $\tau$-equations are defined as usual. Now a $\tau$-term is called strictly positive if it does not contain any negation symbol; positive (negative, resp.) if every variable occurs in the scope of an even (odd, resp.) number of negation symbols; and a boxed variable if it is of the form $f_{\delta}^{1} \ldots f_{\delta}^{n} x(n \geq 0)$, where $x$ is a variable and each $f_{\delta}^{i}$ is the dual of a unary function symbol. A $\tau$-term is untied if it is obtained from boxed variables and negative terms by applying,$+ \cdot$ and the $\tau$-symbols only; we call it weakly untied if the boxed variables are really variables (i.e., $n=0$ in the definition of a boxed variable).

An equation is called strictly positive if there are no occurrences of the negation sign. A Sahlqvist equation is of the form $s \leq t$, where $s$ is untied and $t$ is positive ${ }^{3}$; in a simple Sahlqvist equation, $s$ should be weakly untied.

As an example, consider one of the conjugation axioms, say, $f g_{\delta} x \leq x$. The term $g_{\delta} x$ is a boxed atom, whence $f g_{\delta} x$ is untied; since $x$ is positive, we have a Sahlqvist equation here. For more information on and examples of Sahlqvist equations, the reader is referred to DE RiJke \& Venema [2] (but note that this paper uses a slightly wider definition of a Sahlqvist equation).

All results and definitions in this paper are understood to be indexed by a similarity type $\tau$ mentioning of which will be suppressed from now on.

\footnotetext{
${ }^{2}$ Algebraists tend to write $R_{f} b_{1} \ldots b_{n} a$ instead of $R_{f} a b_{1} \ldots b_{n}$.

${ }^{3}$ The inequality $s \leq t$ is equivalent to the equation $s \cdot-t=0$, and the term $s \cdot-t$ is untied iff $s$ is untied and $t$ is positive. Therefore, Sahqvist equations could equivalently have been defined as being of the form $s=0$, with $s$ untied.
} 


\section{The conjugated case}

In this section we give the proof of Theorem 1. For this aim, let $\mathfrak{A}$ and $\mathfrak{B}$ be two atomic, conjugated BAOs (with the understanding that the conjugated operators of $\mathfrak{A}$ correspond to those of $\mathfrak{B}$ ). Assume that $\mathfrak{F}$ is the common atom structure of $\mathfrak{A}$ and $\mathfrak{B}$, i.e., $\mathfrak{F}=\mathfrak{A} \mathfrak{t} \mathfrak{A}=\mathfrak{A} \mathfrak{t} \mathfrak{B}$.

From Lemma 1 below we may infer that both $\mathfrak{A}$ and $\mathfrak{B}$ can be embedded in the full complex algebra $\mathfrak{C m} \mathfrak{F}$ of $\mathfrak{F}$. In order to apply this lemma, we need the fact that conjugated BAOs are completely additive.

Lemma 1 Let $\mathfrak{C}$ be an atomic, completely additive boolean algebra with operators. Then the map $r: C \rightarrow \mathcal{P}($ Atc $)$ given by

$$
r: c \mapsto\{a \in A t \mathfrak{C} \mid a \leq c\}
$$

preserves infinite joins and embeds $\mathfrak{C}$ into $\mathfrak{C m} \mathfrak{A} \mathfrak{t}$.

Proof. The result that $r$ is an embedding seems to be folklore, (cf. GoldBlatt [3]), while HiRsch \& HodKInson [4] prove that $r$ preserves arbitrary joins. Let us just show here that $r$ is a homomorphism with respect to an arbitrary unary operator $f$, i.e., that

$$
r(f c)=m_{R_{f}}(r(c)) .
$$

First,

$$
r(f c)=r\left(f\left(\sum_{c \geq b \in A t \mathfrak{C}} b\right)\right)=r\left(\sum_{c \geq b \in A t \mathfrak{C}} f b\right),
$$

because $\mathfrak{C}$ is atomic and $f$ is completely additive. Second, since $r$ preserves arbitrary joins, we have that

$$
r\left(\sum_{c \geq b \in A t_{\mathfrak{C}}} f b\right)=\bigcup_{c \geq b \in A t_{\mathfrak{C}}} r(f b) .
$$

Combining (2) and (3) yields:

$$
r(f c)=\{a \in A t \mathfrak{C} \mid \exists b \in A t \mathfrak{C}(b \leq c \& a \in r(f b))\} .
$$

Thus, using the definitions of the map $r$ and the relation $R_{f}$ on Atce we obtain

$$
\begin{aligned}
r(f c) & =\{a \in A t \mathfrak{C} \mid \exists b \in A t \mathfrak{C}(b \in r(c) \& a \leq f b)\} . \\
& =\left\{a \in A t \mathfrak{C} \mid \exists b \in \operatorname{At\mathfrak {C}}\left(b \in r(c) \& R_{f} a b\right)\right\},
\end{aligned}
$$

which by definition of $m_{R_{f}}$ is nothing but (1) itself.

QED

Remark As we mentioned before, Lemma 1 implies that both our conjugated, atomic algebras $\mathfrak{A}$ and $\mathfrak{B}$ embed in the full complex algebra of their common atom structure $\mathfrak{F}$. Now consider the subalgebra of $\mathfrak{C m} \mathfrak{F}$ that is generated by its atoms: a rather easy argument shows that this algebra embeds in both $\mathfrak{A}$ and $\mathfrak{B}$. The embedding into, say, $\mathfrak{A}$, is given by

$$
t_{\mathfrak{C m} \mathfrak{F}}\left(\left\{a_{1}\right\}, \ldots,\left\{a_{n}\right\}\right) \mapsto t_{\mathfrak{A}}\left(a_{1}, \ldots, a_{n}\right),
$$


where $t$ is a term in the algebraic language and $a_{1}, \ldots, a_{n}$ are atoms of $\mathfrak{A}$. In other words, $\mathfrak{A}$ and $\mathfrak{B}$ have both a common subalgebra and a common superalgebra.

Since equational classes are closed under taking subalgebras, it follows immediately from Lemma 1 that any equation holds in both $\mathfrak{A}$ and $\mathfrak{B}$ whenever it is valid in $\mathfrak{C} \mathfrak{F} \mathfrak{F}$. One of the very nice properties of Sahlqvist equations is that their validity also moves the other way around:

Theorem 2 Let $\mathfrak{C}$ be a conjugated atomic boolean algebra with operators. Then for any Sahlqvist equation $\eta$ :

$$
\mathfrak{C}=\eta \Rightarrow \mathfrak{C m} \mathfrak{A} \mathfrak{C}=\eta
$$

Proof. This theorem is an immediate corollary of Theorem 3.5 in VenEma [11]. In order to see why this is so, let us rephrase the content of that theorem in terms of conjugated BAOs . For simplicity, assume that the similarity type contains only one pair of conjugated, unary operators. Let us call a complex algebra over a relational structure $\mathfrak{F}$ discrete if it contains all the singleton sets of states in $\mathfrak{F}$. A tense frame for this similarity type is any relational structure for which the binary relations corresponding to the operations are each other's converse. Now Theorem 3.5 of [11] states that for any discrete complex algebra $\mathfrak{A}$ over a tense frame $\mathfrak{F}$, and any Sahlqvist equation $\eta$, validity of $\eta$ in $\mathfrak{A}$ implies validity of $\eta$ in $\mathfrak{C} \mathfrak{F}$.

Now assume that $\mathfrak{C}$ is a conjugated BAO, and $\eta$ is a Sahlqvist equation such that $\mathfrak{C} \models \eta$. It follows from Lemma 1 that $\mathfrak{C}$ is a discrete complex algebra over the frame $\mathfrak{A} \mathfrak{C}$. It is also rather easy to see that $\mathfrak{A t} \mathfrak{C}$ is in fact a tense frame, since for any two atoms $a, b$ of $\mathfrak{C}$, we have

$$
R_{f} a b \Longleftrightarrow a \leq f(b) \Longleftrightarrow a \cdot f(b) \neq 0 \Longleftrightarrow g(a) \cdot b \neq 0 \Longleftrightarrow b \leq g(a) \Longleftrightarrow R_{g} b a .
$$

But these are precisely the conditions needed to ensure that $\mathfrak{C m} \mathfrak{A} \mathfrak{t}=\eta$.

For readers unfamiliar with the terminology and techniques from modal logic, we treat a simple yet characteristic example here, using algebraic methods only.

Let $f$ and $g$ be conjugated operators on the atomic boolean algebra $(C,+,-, 0)$; denote $\mathfrak{C}=(C,+,-, 0, f, g)$ and $\mathfrak{C}^{\bullet}=\mathfrak{C} \mathfrak{m} \mathfrak{A} \mathfrak{C}$. The operators $m_{R_{f}}$ and $m_{R_{g}}$ of $C^{\bullet}$ will be abbreviated by $f^{\bullet}$ and $g^{\bullet}$, respectively. It is not difficult to verify that $f^{\bullet}$ and $g^{\bullet}$ are conjugates as well; the easiest proof would use the fact that $\mathfrak{A} \mathfrak{C} \mathfrak{C}$ is a tense frame. Note that restricted to the universe $C$ of $\mathfrak{C}, f^{\bullet}$ and $g^{\bullet}$ are just the operators $f$ and $g$ - this is because $\mathfrak{C}$ is a subalgebra of $\mathfrak{C}^{\bullet}$ by Lemma 1. In the sequel, we will write $f c$ instead of $f^{\bullet} c$ once we know that $c$ is an element of $C$, and likewise for $g$.

Now assume that the equation

$$
f f_{\delta} x \leq f_{\delta} f x
$$

holds in $\mathfrak{C}$; we will prove that it holds in $\mathfrak{C}^{\bullet}$ as well.

Let $d$ be an arbitrary element of $\mathfrak{C}^{\bullet}$; since $\mathfrak{C}^{\bullet}$ is atomic it suffices to show that

$$
\forall a \in \operatorname{AtC}^{\bullet}\left(a \leq f^{\bullet} f_{\delta}^{\bullet} d \Rightarrow a \leq f_{\delta}^{\bullet} f^{\bullet} d\right)
$$


Hence, let $a$ be an arbitrary atom of $\mathfrak{C}^{\bullet}$ such that $a \leq f^{\bullet} f_{\delta}^{\bullet} d$. Since $f^{\bullet}$ is completely additive, there is an atom $b$ of $\mathfrak{C}^{\bullet}$ such that $a \leq f^{\bullet} b$ and $b \leq f_{\delta}^{\bullet} d$. Since $\mathfrak{A t} \mathfrak{C}=\mathfrak{A t} \mathfrak{C} \bullet$, the elements $a$, $b$ and $f^{\bullet} b=f b$ actually belong to $C$. Thus, by the conjugacy of $f^{\bullet}$ and $g^{\bullet}, b \leq f_{\delta}^{\bullet} d$ gives us that $g b\left(=g^{\bullet} b\right) \leq d$. This element $g b$ plays an important role in this proof: the essentially trivial but crucial observation about $g b$ is that it too is an element of $C$. Hence, from the assumption that $\gamma$ holds in $\mathfrak{C}$ it follows that

$$
f f_{\delta}(g b) \leq f_{\delta} f(g b) .
$$

By conjugation, $g b \leq g b$ implies that $b \leq f_{\delta} g b$; since $a \leq f b$, this gives $a \leq f f_{\delta} g b$. So, from (5) we may infer

$$
a \leq f_{\delta} f(g b) .
$$

Now we turn to the right hand side, $f_{\delta} f x$, of the Sahlqvist equation, or inequality. The key observation now is that $f_{\delta} f x$ is a positive term. A well-known property of positive terms is that they will be interpreted by monotonic operations. In the particular case of $\mathfrak{C}^{\bullet}$, this amounts to

$$
\mathfrak{C}^{\bullet}=y \leq z \rightarrow f_{\delta}^{\bullet} f^{\bullet} y \leq f_{\delta}^{\bullet} f^{\bullet} z .
$$

But then (with $g b$ for $y$ and $d$ for $z$ ), $g b \leq d$ implies

$$
f_{\delta} f g b \leq f_{\delta}^{\bullet} f^{\bullet} d .
$$

Combining this with (6), we obtain the desired $a \leq f^{\bullet} f_{\delta}^{\bullet} d$. This implies (4).

QED

With the help of Theorem 2 we now have a very simple proof of Theorem 1.

Proof of Theorem 1. If the Sahlqvist equation $\eta$ holds in $\mathfrak{A}$, then by Theorem 2 it holds in $\mathfrak{C m} \mathfrak{F}$. And since $\mathfrak{B}$ is a subalgebra of $\mathfrak{C m} \mathfrak{F}$ by Lemma 1 , we find that $\eta$ is valid in $\mathfrak{B}$. This shows that

$$
\mathfrak{A}=\eta \Rightarrow \mathfrak{B}=\eta .
$$

The converse implication is of course proved in the very same way.

QED

Proof of Corollary 1. The inclusion $\operatorname{Str} V \subseteq A t V$ is immediate from the definitions. For the other direction, let $\mathfrak{F}$ be a relational structure in At $\mathrm{V}$. Then by definition there is an atomic algebra $\mathfrak{A}$ in $\bigvee$ such that $\mathfrak{F}=\mathfrak{A} \mathfrak{t} \mathfrak{A}$. By the assumption that $\mathrm{V}$ is a conjugated Sahlqvist variety, Theorem 2 implies that $\mathfrak{C m} \mathfrak{F}$ belongs to $V$. But then, by definition, $\mathfrak{F}$ is in Str V.

QED

Proof of Corollary 2. The inclusion ' $\subseteq$ ' is immediate by the canonicity of $\vee$. For, let $\mathfrak{A}$ be an algebra in $\mathrm{V}$; by canonicity of $\mathrm{V}$, the canonical embedding algebra $\mathfrak{A}^{\prime}:=\mathfrak{C} \mathfrak{m} \mathfrak{c} \mathfrak{A}$ of $\mathfrak{A}$ is in $V$ as well. But then $\mathfrak{A} \mathfrak{t} \mathfrak{A}^{\prime}(=\mathfrak{C} \mathfrak{s} \mathfrak{A})$ is in At $\mathrm{V}$, whence $\mathfrak{A}^{\prime}=\mathfrak{C} \mathfrak{m} \mathfrak{A} \mathfrak{t} \mathfrak{A}^{\prime}$ is in Cm At $\mathrm{V}$. This implies that $\mathfrak{A}$, being a subalgebra of $\mathfrak{A}^{\prime}$, is in $\mathrm{SCm} A \mathrm{t} V$. The reverse inclusion follows immediately from Corollary 1.

QED 


\section{The non-conjugated case}

Finally, what happens if we drop the conjugation condition from Theorem 1 or the related results? The following Theorem shows that even for strictly positive equations the Theorems 1 and 2 will no longer hold.

Theorem 3 There exist two atomic boolean algebras with operators $\mathfrak{A}^{\prime}$ and $\mathfrak{A} \cong \mathfrak{C m} \mathfrak{A} \mathfrak{t} \mathfrak{A}^{\prime}$, and two strictly positive (and hence, Sahlqvist) equations $\eta_{0}$ and $\eta_{1}$ such that

$$
\begin{aligned}
& \mathfrak{A}^{\prime}=\eta_{0} \quad \text { but } \quad \mathfrak{A} \mid \neq \eta_{0}, \\
& \mathfrak{A}=\eta_{1} \quad \text { but } \quad \mathfrak{A}^{\prime} \not \models \eta_{1} \text {. }
\end{aligned}
$$

Proof. We will work in a similarity type with three unary operator symbols, $f, g$ and $d$; the corresponding operators of $\mathfrak{A}$ and $\mathfrak{A}^{\prime}$ will be presented in the same order.

We start with $\mathfrak{A}$; its boolean part is just the power set algebra of $Z$, the set of all integer numbers. In order to define the operators of $\mathfrak{A}$, let, for a given subset $X \subseteq Z, X^{+}, X^{-}$and $\Delta X$ denote the following sets:

$$
\begin{aligned}
X^{+} & =\{z+1 \mid z \in X\} \\
X^{-} & =\{z-1 \mid z \in X\} \\
\Delta X & = \begin{cases}? & \text { if } X=? \\
Z \backslash\{z\} & \text { if } X=\{z\} \\
Z & \text { otherwise. }\end{cases}
\end{aligned}
$$

Then $\mathfrak{A}$ is simply defined as the algebra

$$
\mathfrak{A}:=\left(\mathcal{P}(Z), \cup,-, ?,(\cdot)^{+},(\cdot)^{-}, \Delta\right) .
$$

It is easy to verify that $(\cdot)^{+},(\cdot)^{-}$and $\Delta$ are operators. In fact, we may observe that $X^{+}=$ $m_{P}(X), X^{-}=m_{S}(X)$ and $\Delta X=m_{\neq}(X)$, where $P, S$ and $\neq$ are the predecessor relation $(y P z$ iff $y=z+1)$, the successor relation and the inequality relation, respectively. Hence, $\mathfrak{A}$ is a full complex algebra:

$$
\mathfrak{A}=\mathfrak{C} \mathfrak{m}(Z, P, S, \neq) .
$$

The definition of $\mathfrak{A}^{\prime}$ is more involved — we need some auxiliary definitions first. A set $X \subseteq Z$ is called cofinite if its complement (wrt $Z$ ) is finite; we denote by $\mathcal{P}^{*}(Z$ ) the collection of finite and cofinite subsets of integers. Then, $X \oplus Y$ denotes the symmetric difference of $X$ and $Y$, i.e., $X \oplus Y=(X \backslash Y) \cup(Y \backslash X)$. E and $O$ denote the sets of all even and all odd numbers, respectively. Now we define $A^{\prime}$, the carrier set of $\mathfrak{A}^{\prime}$ :

$$
A^{\prime}=\left\{X \subseteq Z \mid X \in \mathcal{P}^{*}(Z) \text { or } E \oplus X \in \mathcal{P}^{*}(Z)\right\} .
$$

It may be useful to note that $-(E \oplus X)=O \oplus X$ and that

$$
X \in A^{\prime} \text { iff one of: } X,-X, E \oplus X \text { or } O \oplus X \text {, is finite. }
$$

These two observations may facilitate the rather tedious (but not very difficult) proof that $A^{\prime}$ is closed under taking unions and complements - the actual proof is left to the reader. 
Now consider the following operations on $\mathcal{P}(Z)$ :

$$
\begin{aligned}
& X^{>}= \begin{cases}X^{+} & \text {if either } X \text { or } E \oplus X \text { is finite } \\
Z & \text { otherwise }\end{cases} \\
& X^{<}= \begin{cases}X^{-} & \text {if either } X \text { or } O \oplus X \text { is finite } \\
Z & \text { otherwise. }\end{cases}
\end{aligned}
$$

In other words, $X^{>}=X^{+}$iff $X$ is 'almost everywhere' the same as either ? or $E$. It is quite easy to show that $A^{\prime}$ is closed under the three operations just defined; hence, the following correctly defines an algebra (the operation $\Delta$ is as defined above):

$$
\mathfrak{A}^{\prime}:=\left(A^{\prime}, \cup,-, ?,(\cdot)^{>},(\cdot)^{<}, \Delta\right) .
$$

On the other hand, it is another quite tedious task to check that $\mathfrak{A}^{\prime}$ is a BAO. For instance, in order to check the additivity of $(\cdot)^{>}$, we need to show that for arbitrary sets of integers $X$ and $Y$ in $A^{\prime}$ :

$$
(X \cup Y)^{>}=X^{>} \cup Y^{>} .
$$

The proof of (8) proceeds by distinguishing cases, as to the nature of $X$ and $Y$ with respect to (7). We only treat one example here: suppose that $E \oplus X$ and $O \oplus Y$ are finite. In other words, there are only finitely many places where $X$ differs from $E$, and likewise with $Y$ and $O$. But then surely $X \cup Y$ must be a cofinite set. Thus

$$
(X \cup Y)^{>}=Z=X^{+} \cup Z=X^{>} \cup Y^{>} .
$$

This indicates how to prove (8).

$\mathfrak{A}^{\prime}$ is clearly atomic: its set of atoms consists of the singleton subsets of $Z$. In other words, $A t \mathfrak{A}^{\prime}=$ At $\mathfrak{A}$. Now take an arbitrary atom $\{z\}$; we have $\{z\}^{>}=\{z\}^{+}$since $\{z\}$ is finite; likewise, $\{z\}^{<}=\{z\}^{-}$. Hence, $\mathfrak{A} \mathfrak{t} \mathfrak{A}^{\prime}=\mathfrak{A} \mathfrak{t} \mathfrak{A}$, and since $\mathfrak{A}$ is a full complex algebra, we have now established that $\mathfrak{A}^{\prime}$ and $\mathfrak{A}$ meet the first requirements of the theorem:

$\mathfrak{A}$ and $\mathfrak{A}^{\prime}$ are atomic BAOs such that $\mathfrak{A} \cong \mathfrak{C} \mathfrak{m} \mathfrak{A} \mathfrak{A} \mathfrak{A}^{\prime}$.

We will now discuss a strictly positive equation that is valid in $\mathfrak{A}$, but not in $\mathfrak{A}^{\prime}$. To be more precise, we will show that

$$
\mathfrak{A} \models f g x \leq g f x, \text { while } \mathfrak{A}^{\prime} \not \models f g x \leq g f x .
$$

First we show that the equation is valid in $\mathfrak{A}$; let $X$ be an arbitrary subset of $\mathfrak{A}$, then

$$
f_{\mathfrak{A}} g_{\mathfrak{A}}(X)=\left(X^{-}\right)^{+}=X \subseteq X=\left(X^{+}\right)^{-}=g_{\mathfrak{A}} f_{\mathfrak{A}}(X) .
$$

On the other hand, if we consider the element $E$ in $\mathfrak{A}^{\prime}$, we find:

$$
f_{\mathfrak{A}^{\prime}} g_{\mathfrak{A}^{\prime}}(E)=\left(E^{<}\right)^{>}=Z^{>}=Z,
$$

while

$$
g_{\mathfrak{A}^{\prime}} f_{\mathfrak{A}^{\prime}}(E)=\left(E^{>}\right)^{<}=\left(E^{+}\right)^{<}=O^{<}=O^{-}=E .
$$


This proves (9). Finally, we will show that conversely, there is a strongly positive equation which is valid in $\mathfrak{A}^{\prime}$, but not in $\mathfrak{A}$ :

$$
\mathfrak{A}^{\prime} \models d x \leq f d x, \text { while } \mathfrak{A} \not \models d x \leq f d x .
$$

First consider $\mathfrak{A}$ : if we take the singleton $\{1\}$ for $x$, the left hand side of the inequality adds up to the set $Z \backslash\{1\}$, while the right hand side yields the set $(\Delta\{1\})^{+}=(Z \backslash\{1\})^{+}=Z \backslash\{2\}$; this shows the inequality not to hold in $\mathfrak{A}$.

Now, turning to $\mathfrak{A}^{\prime}$, let $X$ be an arbitrary element of $A^{\prime}$. Distinguish the following cases:

(a) $X=$ ? Then both sides of the inequality give the empty set.

(b) $X=\{z\}$ for some integer $z$. It suffices to show that the right hand side (rhs) of the inequality will amount to the top set $Z$ of the algebra:

$$
f_{\mathfrak{A}^{\prime}} d_{\mathfrak{A}^{\prime}}(X)=(\Delta\{z\})^{>}=(Z \backslash\{z\})^{>}=Z .
$$

(c) $|X|>1$. Again, the rhs gives $Z$ :

$$
f_{\mathfrak{A}^{\prime}} d_{\mathfrak{A}^{\prime}}(X)=(\Delta X)^{>}=Z^{>}=Z .
$$

This proves (10), and hence, finishes the proof of Theorem 3.

QED

Note that the algebras $\mathfrak{A}$ and $\mathfrak{A}^{\prime}$ defined in the just given proof also constitute a counterexample to a 'non-completely-additive version' of Lemma 1: although $\mathfrak{A}=\mathfrak{C m} \mathfrak{A} \mathfrak{t} \mathfrak{A}^{\prime}$, there is no way to embed $\mathfrak{A}^{\prime}$ in $\mathfrak{A}$. This observation might lead one to wonder whether an analogue of Theorem 1 does hold when $\mathfrak{A}$ and $\mathfrak{B}$ are subalgebras of the full complex algebra of their common atom structure. This is not the case, as the following Theorem shows.

Theorem 4 There exist completely additive, atomic boolean algebras with operators $\mathfrak{B}^{\prime}$ and $\mathfrak{B}=\mathfrak{C} \mathfrak{m} \mathfrak{A} \mathfrak{\mathfrak { B } ^ { \prime }}$ with $\mathfrak{B}^{\prime}$ a subalgebra of $\mathfrak{B}$, and a Sahlqvist equation $\gamma$ such that

$$
\mathfrak{B}^{\prime} \models \gamma \text { but } \mathfrak{B} \not \models \gamma .
$$

Proof. This Theorem is an immediate consequence of Lemma 6.7 in Venema [11]. For, consider the relational structure $\mathfrak{F}=(W, R)$ which is as in Figure 1. To be more precise, the set of states is given as $W=\{u, v, w, x\} \cup\left\{v_{n}, w_{n} \mid n \in \omega\right\}$, and the relation $R$ holds as follows: Ruv, Ruw, Rvv $v_{n}$ and $R w w_{n}$, all $n, R v_{n} x$ and $R w_{n} x$, all $n$, and $R x x$.

Now $\mathfrak{B}$ is defined as the full complex algebra of the structure $\mathfrak{F}$, and $\mathfrak{B}^{\prime}$ is the subalgebra generated by the atoms of $\mathfrak{B}$. Finally, $\gamma$ is the formula $f f_{\delta} x \leq f_{\delta} f x$ (precisely the example we treated in the proof of Theorem 2). Details of the proof that $\gamma$ holds in $\mathfrak{B}^{\prime}$, but not in $\mathfrak{B}$, can be found in the proof of Lemma 6.7 in the cited paper.

QED

One may now wonder under which conditions a non-conjugated version of Theorem 2 does hold. Here is a positive result: 


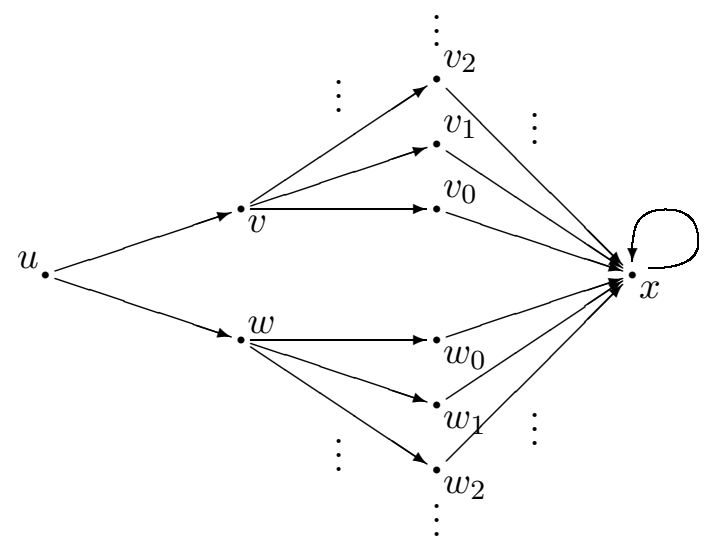

Figure 1: Atom structure.

Theorem 5 Let $\mathfrak{C}$ be a completely additive, atomic BAO. Then for every simple Sahlqvist equation $\eta$ :

$$
\mathfrak{C} \mid=\eta \text { iff } \mathfrak{C} \mathfrak{m} \mathfrak{A} \mathfrak{c} \mid=\eta \text {. }
$$

Proof. For the direction from right to left we apply Lemma 1 to ensure that $\mathfrak{C}$ is a subalgebra of $\mathfrak{C m} \mathfrak{A} \mathfrak{C}$. The other direction is, just like Theorem 2, an immediate consequence of Theorem 3.5 in Venema [11]. For, in the context of ordinary (not necessarily conjugated) BAOs, a tense frame is just any ordinary relational structure for the similarity type, and a Sahlqvist tense formula is the modal correspondent of a simple Sahlqvist formula. Obviously, one could also give a rather easy algebraic proof for this direction, in the same style as our example in the proof of Theorem 2 .

QED

Thus, we seem to have a fairly complete answer to the question for which atomic BAOs $\mathfrak{A}$ and which Sahlqvist equations $\eta$ we have that $\mathfrak{A}=\eta$ implies $\mathfrak{C m} \mathfrak{A} \mathfrak{A}=\eta$ : if $\mathfrak{A}$ is conjugated and $\eta$ is any Sahlqvist equation, or if $\mathfrak{A}$ is completely additive and $\eta$ is any simple Sahlqvist equation, then the answer is positive. For the other cases we have established counterexamples.

\section{References}

[1] H. Andréka. On the complexity of the equations valid in algebras of relations, 1991. Thesis for D.Sc. (a post-habilitation degree) with Math. Inst. Hungar. Ac. Sci. Budapest. A slightly updated version will appear in Annals of Pure and Applied Logic.

[2] M. de Rijke and Y. Venema. Sahlqvist's theorem for Boolean algebras with operators. Studia Logica, 54:61-78, 1995.

[3] R. Goldblatt. Elementary generation and canonicity for varieties of boolean algebras with operators. Algebra Universalis, to appear. 
[4] R. Hirsch and I. Hodkinson. Complete representations in algebraic logic. Technical report, Department of Computing, Imperial College, London, 1994. To appear in Journal of Symbolic Logic.

[5] I. Hodkinson. Atom structures of cylindric algebras and relation algebras. Technical report, Department of Computing, Imperial College, London, 1997. To appear in Annals of Pure and Applied Logic.

[6] B. Jónsson. On the canonicity of Sahlqvist identities. Studia Logica, 53:473-491, 1995.

[7] B. Jónsson and A. Tarski. Boolean algebras with operators. Parts I and II. American Journal of Mathematics, 73:891-939, 1951. and 74:127-162, 1952.

[8] R. D. Maddux. Introductory course on relation algebras. In H. Andréka, J. D. Monk, and I. Németi, editors, Algebraic Logic (Proc. Conf. Budapest 1988), pages 361-392, 1991.

[9] J. D. Monk. Nonfinitizability of classes of representable cylindric algebras. Journal of Symbolic Logic, 34:331-343, 1969.

[10] J. D. Monk. Completions of Boolean algebras with operators. Mathematische Nachrichten, 46:47-55, 1970.

[11] Y. Venema. Derivation rules as anti-axioms in modal logic. Journal of Symbolic Logic, 58:1003-1034, 1993.

[12] Y. Venema. Atom structures and Sahlqvist equations. Research Report 96-173, Mathematics Department, Victoria University of Wellington, 1996.

[13] Y. Venema. Atom structures. In M. Kracht, M. de Rijke, H. Wansing, and M. Zakharyaschev, editors, Advances in Modal Logic '96, Stanford, 1997. CSLI Publications. 